\title{
Territory wide study of patients with dystrophinopathy in Hong Kong
}

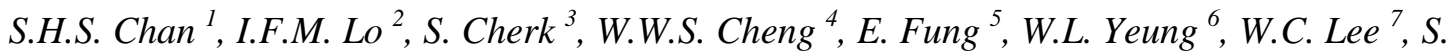 \\ Wong $^{8}$, C.K.L. Ma ${ }^{8}$, S.M. $\mathrm{Tai}^{9}$, M.M. Yau ${ }^{10}$, G. $\mathrm{Ng}^{11}$, S.P. Wu ${ }^{12}$, C.V.N. Wong ${ }^{1}$ \\ ${ }^{1}$ Dept of Paediatrics and Adolescent Medicine, Queen Mary Hospital, Hong Kong; ${ }^{2}$ Clinical \\ Genetic Service, Dept of Health, Hong Kong; ${ }^{3}$ Dept of Paediatrics and Adolescent Medicine, \\ Kwong Wah Hospital, Hong Kong; ${ }^{4}$ Dept of Paediatrics and Adolescent Medicine, Caritus \\ Medical Centre, Hong Kong; ${ }^{5}$ Dept of Paediatrics and Adolescent Medicine, Prine of Wales \\ Hospital, Hong Kong; ${ }^{6}$ Dept of Paediatrics and Adolescent Medicine, Alice Ho Mei Ling \\ Nethesole Hospital, Hong Kong; ${ }^{7}$ Dept of Paediatrics and Adolescent Medicine, Tuen Mun \\ Hospital, Hong Kong $;^{8}$ Dept of Paediatrics and Adolescent Medicine, United Christian Hospital, \\ Hong Kong; ${ }^{9}$ Dept of Paediatrics and Adolescent Medicine, Pamela Youde Nethesole Eastern \\ Hospital, Hong Kong; ${ }^{10}$ Dept of Paediatrics and Adolescent Medicine, Tseung Kwan O Hospital, \\ Hong Kong; ${ }^{I I}$ Dept of Paediatrics and Adolescent Medicine, Princess Margaret Hospital, Hong \\ Kong; ${ }^{12}$ Dept of Paediatrics and Adolescent Medicine, Queen Elizabeth Hospital, Hong Kong
}

This is a first territory wide study in Hong Kong on Chinese patients with dystrophinopathy on their genetic mutation, motor performance, use of steroid, and the interventions they received. This study is participated by all the paediatric departments in the eleven hospitals in Hong Kong. Clinical data was systemically collected from medical records of all patients diagnosed dystrophinopathy and were actively followed-up between May 2006 and April 2010. We found 91 individuals with dystrophinopathy, 82\% have Duchenne Muscular Dystrophy (DMD) and 18\% have Becker Muscular Dystrophy (BMD). The overall prevalence of dystrophinopathy, for 2010, is 1.03 per 10,000 males aged $0-24$ years old. Mutation consists of $47 \%$ of large deletion and $10 \%$ of large duplication, and $43 \%$ of small rearrangement or point mutation, of which $59.4 \%$ are nonsense mutations. Exon deletion in the distal hot spot (exons 45-55) represent up to 54\% of the identified deletion, while deletion starting in the proximal hot spot (exon 2-20) account for 37\%. Only $23 \%$ of children had been on steroid and all stopped before or when they lost ambulation. For DMD individuals, the age ranges from 0.5 to 34 years old, with $48 \%$ are older than 13 years old, and the mean age of loss of ambulation is 10.5 years old. For those older than 13 years old, $30 \%$ have cardiomyopathy, 19\% required noninvasive ventilation, $15 \%$ had scoliosis surgery and only $5 \%$ had gastrostomy. This first territory wide study for individuals with dystrophinopathy in Hong Kong confirms a similar prevalence of such condition with the western countries, but our population has a much higher percentage of point mutation. The findings of infrequent steroid use and the low percentage of gastrostomy among patients with noninvasive ventilation, allows us to compare our current approaches in Hong Kong with the DMD care guidelines to drive improvements in our health care delivery. 